On the global and weak dimensions of pullbacks of non-commutative rings 


\title{
On the global and weak dimensions of pullbacks of non-commutative rings
}

\author{
Nikolai Kosmatov
}

All rings will be assumed to have identity elements preserved by ring homomorphisms, and all modules, unless specified otherwise, will be left modules. For a ring $S$, $\operatorname{lgld} S$ and lwd $S$ will denote the left global dimension of $S$ and the left weak global dimension of $S$, respectively. For an $S$-module $X$ and a right $S$-module $Y$, the projective dimension of $X$, the injective dimension of $X$, the flat dimension of $X$, and the flat dimension of $Y$ are denoted by $\operatorname{pd}_{S} X, \mathrm{id}_{S} X, \mathrm{fd}_{S} X$ and $\operatorname{rdd}_{S} Y$, respectively.

A commutative square of rings and ring homomorphisms

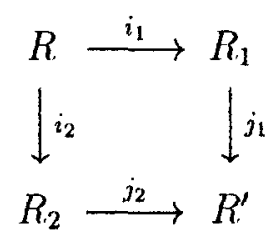

is said to be a pullback (or a cartesian square, or a fibre product) if given $r_{1} \in R_{1}, r_{2} \in R_{2}$ with $j_{1}\left(r_{1}\right)=j_{2}\left(r_{2}\right)$ there is a unique element $r \in R$ such that $i_{1}(r)=r_{1}$ and $i_{2}(r)=r_{2}$ (note that if $j_{2}$ is a surjection then so is $i_{1}$, but not conversely). The ring $R$ is called the fibre product (or pullback) of $R_{1}$ and $R_{2}$ over $R^{\prime}$.

Assuming that $j_{2}$ is surjective, Milnor [5, Chapter 2] characterized projective modules over such a ring $R$. Facchini and Vámos [2] established analogues of Milnor's theorems for injective and flat modules. Kirkman and Kuzmanovich [3, Theorem 2] showed that if (1) is a pullback square with $j_{2}$ surjective, then

$$
\operatorname{lgld} R \leqslant \max _{k=1,2}\left\{\operatorname{lgld} R_{k}+\operatorname{rfd}_{R} R_{k}\right\}
$$

For commutative rings, Scrivanti $[G]$ sharpened this upper bound on Igld $R$ and obtained an upper bound on lwd $R$. Moreover, she gave examples which 
showed that, in a certain case, those results were best possible. Besides, Cowley showed that it could be beneficial to work only on one side of the rings in question [1, Example 3.4]. He obtained the following "one-sided" bound [1, Theorem 3.1]: if (1) is a pullback square with $j_{2}$ surjective, then

$$
\operatorname{lgld} R \leqslant \max _{k=1,2}\left\{\operatorname{lgld} R_{k}+\operatorname{pd}_{R} R_{k}\right\} .
$$

Our Theorems 9,10 and Corollaries 12,13 are the generalizations to non-commutative rings of Scrivanti's results. In Theorem 8 , we provide a "one-sided" bound on the left global dimension of a pullback ring. In Propositions 5,6 and 7, we give sufficient conditions for an $R$-module $M$ to have injective, projective and flat dimensions $\leqslant n$. In Corollaries 11 and 12 , we deduce the upper bounds (3) and (2) as immediate consequences of our Propositions 5 and 6 . Here we relax the conditions and only require $i_{1}$ to be surjective.

We begin with the following consequence of [2, Theorem 2].

Theorem 1. Let (1) be a pullback diagram with $i_{1}$ surjective. Then an $R$-module $M$ is injective (projective, flat) if and only if $\operatorname{Hom}_{R}\left(R_{1}, M\right)$ and $\operatorname{Hom}_{R}\left(R_{2}, M\right)\left(R_{1} \otimes_{R} M\right.$ and $\left.R_{2} \otimes_{R} M\right)$ are $R_{1}$ - and $R_{2}$-injective (projective, flat) modules respectively.

Proof. Set $R^{\prime \prime}=j_{2}\left(R_{2}\right)$. Since $i_{1}$ is a surjection, we obtain $j_{1}\left(R_{1}\right) \subset j_{2}\left(R_{2}\right)=R^{\prime \prime}$. Thus we have another commutative square of rings and ring homomorphisms

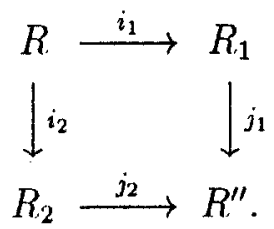

It is clear that this diagram is a pullback square with $j_{2}: R_{2} \rightarrow R^{\prime \prime}$ surjective. So the desired result follows from [2, Theorem 2].

Proposition 2. Let $M$ be an R-module, $n$ be a positive integer, and let

$$
0 \longrightarrow M \stackrel{f_{0}}{\longrightarrow} I_{0} \stackrel{f_{1}}{\longrightarrow} I_{1} \stackrel{f_{2}}{\longrightarrow} I_{2} \longrightarrow \ldots
$$

be an injective resolution of $M$. Let $K_{t}$ denote $\operatorname{im}\left(f_{t+1}\right), t \geqslant 0$. Suppose that $\operatorname{id}_{R_{k}}\left(\operatorname{Ext}_{R}^{l}\left(R_{k}, M\right)\right) \leqslant n-l$ for $l=0,1, \ldots, n$ and $k=1,2$. Then $\operatorname{id}_{R_{k}}\left(\operatorname{Hom}_{R}\left(R_{k}, K_{t}\right)\right) \leqslant n-t-1$ for $t=0,1, \ldots, n$ and $k=1,2$. 
Proof. For any $n \geqslant 1$ and $k=1,2$, the proof is by induction on $t$.

For $t=0$, if we apply the functor $\operatorname{Ext}_{R}^{*}\left(R_{k},-\right)$ to the short exact sequence of $R$-modules $0 \longrightarrow M \longrightarrow I_{0} \stackrel{f_{1}}{\longrightarrow} K_{0} \longrightarrow 0$, we obtain an exact sequence of $R_{k}$-modules

$$
\begin{aligned}
& 0 \longrightarrow \operatorname{Hom}_{R}\left(R_{k}, M\right) \longrightarrow \operatorname{Hom}_{R}\left(R_{k}, I_{0}\right) \stackrel{f_{1 *}}{\longrightarrow} \\
& \stackrel{f_{1 *}}{\longrightarrow} \operatorname{Hom}_{R}\left(R_{k}, K_{0}\right) \longrightarrow \operatorname{Ext}_{R}^{1}\left(R_{k}, M\right) \longrightarrow 0
\end{aligned}
$$

and isomorphisms of $R_{k}$-modules

$$
\operatorname{Ext}_{R}^{l}\left(R_{k}, K_{0}\right) \simeq \operatorname{Ext}_{R}^{l+1}\left(R_{k}, M\right), l \geqslant 1
$$

Set $A_{k, 0}=\operatorname{im} f_{1 *}$ and break up (4) into two short exact sequences of $R_{k}$-modules:

$$
\begin{gathered}
0 \longrightarrow \operatorname{Hom}_{R}\left(R_{k}, M\right) \longrightarrow \operatorname{Hom}_{R}\left(R_{k}, I_{0}\right) \stackrel{f_{1}}{\longrightarrow} A_{k, 0} \longrightarrow 0 \\
0 \longrightarrow A_{k, 0} \hookrightarrow \operatorname{Hom}_{R}\left(R_{k}, K_{0}\right) \longrightarrow \operatorname{Ext}_{R}^{1}\left(R_{k}, M\right) \longrightarrow 0
\end{gathered}
$$

Since $I_{0}$ is an injective $R$-module, it can easily be checked that $R_{k}$-module $\operatorname{Hom}_{R}\left(R_{k}, I_{0}\right)$ is injective. Since $\operatorname{id}_{R_{k}}\left(\operatorname{Hom}_{R}\left(R_{k}, M\right)\right) \leqslant n$, we obtain from (6) that $\operatorname{id}_{R_{k}}\left(A_{k, 0}\right) \leqslant n-1$. At the same time $\operatorname{id}_{R_{k}}\left(\operatorname{Ext}_{R}^{1}\left(R_{k}, M\right)\right) \leqslant n-1$. Therefore, using (7), we get id $R_{k}\left(\operatorname{Hom}_{R}\left(R_{k}, K_{0}\right)\right) \leqslant n-1$.

For $t \geqslant 1$, we apply the functor $\operatorname{Ext}_{R}^{*}\left(R_{k},-\right)$ to the short exact sequence of $R$-modules $0 \longrightarrow K_{t-1} \hookrightarrow I_{t} \stackrel{f_{t+1}}{\longrightarrow} K_{t} \longrightarrow 0$. We get an exact sequence of $R_{k}$-modules

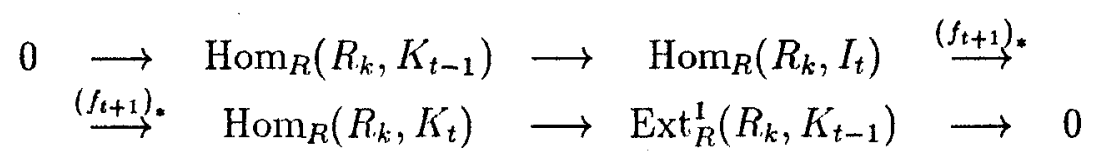

and isomorphisms of $R_{k}$-modules

$$
\operatorname{Ext}_{R}^{l}\left(R_{k}, K_{t}\right) \simeq \operatorname{Ext}_{R}^{l+1}\left(R_{k}, K_{t-1}\right), l \geqslant 1 .
$$

Put $A_{k, t}=\operatorname{im}\left(f_{t+1}\right)_{*}$ and break up (8) into two short exact sequences of $R_{k}$-modules:

$$
\begin{gathered}
0 \longrightarrow \operatorname{Hom}_{R}\left(R_{k}, K_{t-1}\right) \longrightarrow \operatorname{Hom}_{R}\left(R_{k}, I_{t}\right) \stackrel{\left(f_{t+1}\right) *}{\longrightarrow} A_{k, 0} \longrightarrow 0 \\
0 \longrightarrow A_{k, t} \hookrightarrow \operatorname{Hom}_{R}\left(R_{k}, K_{t}\right) \longrightarrow \operatorname{Ext}_{R}^{1}\left(R_{k}, K_{t-1}\right) \longrightarrow 0
\end{gathered}
$$


By the inductive hypothesis, we have $\mathrm{id}_{R_{k}}\left(\operatorname{Hom}_{R}\left(R_{k}, K_{t-1}\right)\right) \leqslant n-t$. Since $I_{t}$ is an injective $R$-module, $\operatorname{Hom}_{R}\left(R_{k}, I_{t}\right)$ is an injective $R_{k}$-module. Therefore, from (10), $\mathrm{id}_{R_{k}}\left(A_{k, t}\right) \leqslant n-t-1$. Combining (5) and (9), we have $\operatorname{Ext}_{R}^{1}\left(R_{k}, K_{t-1}\right) \simeq \ldots \simeq \operatorname{Ext}_{R}^{t}\left(R_{k}, K_{0}\right) \simeq \operatorname{Ext}_{R}^{t+1}\left(R_{k}, M\right)$. Hence $\operatorname{id}_{R_{k}}\left(\operatorname{Ext}_{R}^{1}\left(R_{k}, K_{t-1}\right)\right)=\mathrm{id}_{R_{k}}\left(\operatorname{Ext}_{R}^{t+1}\left(R_{k}, M\right)\right) \leqslant n-t-1$. Finally, from (11), we obtain $\operatorname{id}_{R_{k}}\left(\operatorname{Hom}_{R}\left(R_{k}, K_{t}\right)\right) \leqslant n-t-1$, as required.

The proofs of the following Propositions 3 and 4 are similar to that of Proposition 2 if we apply the functors $\operatorname{Tor}_{*}^{R}\left(R_{k},-\right)$ to the given resolutions.

Proposition 3. Let $M$ be an R-module, $n$ be a positive integer, and let

$$
\cdots \longrightarrow P_{2} \stackrel{f_{2}}{\longrightarrow} P_{1} \stackrel{f_{1}}{\longrightarrow} P_{0} \stackrel{f_{0}}{\longrightarrow} M \longrightarrow 0
$$

be a projective resolution of $M$. Let $K_{t}$ denote ker $f_{t}, t \geqslant 0$. Suppose that $\operatorname{pd}_{R_{k}}\left(\operatorname{Tor}_{l}^{R}\left(R_{k}, M\right)\right) \leqslant n-l$ for $l=0,1, \ldots, n$ and $k=1,2$. Then $\operatorname{pd}_{R_{k}}\left(R_{k} \otimes_{R} K_{t}\right) \leqslant n-t-1$ for $t=0,1, \ldots, n$ and $k=1,2$.

Proposition 4. Let $M$ be an $R$-module, $n$ be a positive integer, and let

$$
\ldots \longrightarrow F_{2} \stackrel{f_{2}}{\longrightarrow} F_{1} \stackrel{f_{1}}{\longrightarrow} F_{0} \stackrel{f_{0}}{\longrightarrow} M \longrightarrow 0
$$

be a flat resolution of $M$. Let $K_{t}$ denote ker $f_{t}, t \geqslant 0$. Suppose that $\mathrm{fd}_{R_{k}}\left(\operatorname{Tor}_{l}^{R}\left(R_{k}, M\right)\right) \leqslant n-l$ for $l=0,1, \ldots, n$ and $k=1,2$. Then $\mathrm{fd}_{R_{k}}\left(R_{k} \otimes R K_{t}\right) \leqslant n-t-1$ for $t=0,1, \ldots, n$ and $k=1,2$.

Proposition 5. Let (1) be a pullback diagrarn with $i_{1}$ surjective, $M$ be an $R$-module, and let $n$ be a non-negative integer. Suppose that $\operatorname{id}_{R_{k}}\left(\operatorname{Ext}_{R}^{l}\left(R_{k}, M\right)\right) \leqslant n-l$ for $l=0,1, \ldots, n$ and $k=1,2$. Then $\operatorname{id}_{R} M \leqslant n$.

Proof. For the case $n=0$, the result follows from Theorem 1 . For $n \geqslant 1$, consider an injective resolution of $R$-module $M$

$$
0 \longrightarrow M \stackrel{f_{0}}{\longrightarrow} I_{0} \stackrel{f_{1}}{\longrightarrow} I_{1} \stackrel{f_{2}}{\longrightarrow} I_{2} \longrightarrow \ldots
$$

By definition, put $K_{t}=\operatorname{im}\left(f_{t+1}\right)$ for $t \geqslant 0$. From Proposition 1 , we get that $R_{k}$-module $\operatorname{Hom}_{R}\left(R_{k}, K_{n-1}\right)$ is injective $(k=1,2)$. Hence, by Theorem 1 , $K_{n-1}$ is an injective $R$-module. This means that $\operatorname{id}_{R} M \leqslant n$.

Arguing as above, the reader will easily prove the following analogues of Proposition 5 for projective and flat dimensions. 
Proposition 6. Let (1) be a pullback diagram with $i_{1}$ surjective, $M$ be an $R$-module, and let $n$ be a non-negative integer. Suppose that $\operatorname{pd}_{R_{k}}\left(\operatorname{Tor}_{l}^{R}\left(R_{k}, M\right)\right) \leqslant n-l$ for $l=0,1, \ldots, n$ and $k=1,2$. Then $\operatorname{pd}_{R} M \leqslant n$.

Proposition 7. Let (1) be a pullback diagram with $i_{1}$ surjective, $M$ be an $R$-module, and let $n$ be a non-negative integer. Suppose that $\mathrm{fd}_{R_{k}}\left(\operatorname{Tor}_{l}^{R}\left(R_{k}, M\right)\right) \leqslant n-l$ for $l=0,1, \ldots, n$ and $k=1,2$. Then $\mathrm{fd}_{R} M \leqslant n$.

Proposition 5 clearly implies the following theorem.

Theorem 8. Let (1) be a pullback diagram with $i_{1}$ surjective, and let $n$ be a non-negative integer. Suppose that for any $R$-module $M$ we have that

$$
\mathrm{id}_{R_{k}}\left(\operatorname{Ext}_{R}^{l}\left(R_{k}, M\right)\right) \leqslant n-l \text { for } l=0,1, \ldots, n \text { and } k=1,2 \text {. }
$$

Then $\operatorname{lgld} R \leqslant n$.

Theorem 9. Let (1) be a pullback diagram with $i_{1}$ surjective, and let $n$ be $a$ non-negative integer. Suppose that for any left ideal $J$ of $R$ we have that

$$
\operatorname{pd}_{R_{k}}\left(\operatorname{Tor}_{l}^{R}\left(R_{k}, R / J\right)\right) \leqslant n-l \text { for } l=0,1, \ldots, n \text { and } k=1,2 .
$$

Then $\operatorname{lgld} R \leqslant n$.

Proof. Let $J$ be a left ideal of $R$. Proposition 6 shows immediately that $\operatorname{pd}_{R}(R / J) \leqslant n$. Therefore, using Auslander's theorem, we have $\operatorname{lgld} R=\sup \left\{\operatorname{pd}_{R}(R / J) \mid J\right.$ is a left ideal of $\left.R\right\} \leqslant n$.

Theorem 10. Let (1) be a pullback diagram with $i_{1}$ surjective, and let $n$ be a non-negative integer. Suppose that for any finitely generated left ideal $J$ of $R$ we have that

$$
\operatorname{fd}_{R_{k}}\left(\operatorname{Tor}_{l}^{R}\left(R_{k}, R / J\right)\right) \leqslant n-l \text { for } l=0,1, \ldots, n \text { and } k=1,2 .
$$

Then lwd $R \leqslant n$.

Proof. Let $J$ be a finitely generated left ideal of $R$. Proposition 7 evidently implies that $\mathrm{fd}_{R}(R / J) \leqslant n$. Therefore, since lwd $R=\sup \left\{\operatorname{pd}_{R}(R / J) \mid J\right.$ is a finitely generated left ideal of $\left.R\right\}$, we have $\operatorname{lgld} R \leqslant n$. 
Corollary 11. If (1) is a pullback diagram with $i_{1}$ surjective, then

$$
\operatorname{lgld} R \leqslant \max _{k=1,2}\left\{\operatorname{lgld} R_{k}+\operatorname{pd}_{R} R_{k}\right\} .
$$

Proof. Set $n_{k}=\operatorname{lgld} R_{k}, m_{k}=\operatorname{pd}_{R} R_{k}, N_{k}=n_{k}+m_{k}(k=1,2)$ and $N=\max \left\{N_{1}, N_{2}\right\}$. It can be assumed that $m_{k}, n_{k}<\infty$. Let $M$ be an $R$-module and $k \in\{1,2\}$. Since $\operatorname{pd}_{R} R_{k}=m_{k}$, we have $\operatorname{Ext}_{R}^{l}\left(R_{k}, M\right)=0$ for all $l \geqslant m_{k}+1$. At the same time, since $\operatorname{lgld} R_{k}=n_{k}$, we get $\operatorname{id}_{R_{k}}\left(\operatorname{Ext}_{R}^{l}\left(R_{k}, M\right)\right) \leqslant n_{k}=N_{k}-m_{k} \leqslant N_{k}-l \leqslant N-l$ for any $l=0,1, \ldots, m_{k}$. Therefore, by Proposition $5, \operatorname{pd}_{R} M \leqslant N$. This means that lgld $R \leqslant N$.

Similarly, Propositions 6 and 7 allow us to prove Corollaries 12 and 13 .

Corollary 12. If (1) is a pulluack diagram with $i_{1}$ surjective, then

$$
\operatorname{lgld} R \leqslant \max _{k=1,2}\left\{\operatorname{lgld} R_{k}+\operatorname{rfd} R_{R}\right\} .
$$

Corollary 13. If (1) is a pullback diagram with $i_{1}$ surjective, then

$$
\operatorname{lwd} R \leqslant \max _{k=1,2}\left\{\operatorname{lwd} R_{k}+\operatorname{rfd}_{R} R_{k}\right\} .
$$

Acknowledgement. The authour would like to thank Professor A. I. Generalov for his help and guidance.

\section{References}

[1] K. M. Cowley. One-sided bounds and the vanishing of Ext. J. Algebra, 190 (1997), 361-371.

[2] A. FACCHINI, P. VÁmos. Injective modules over pullbacks. J. London Math. Soc., 31 (1985), 425-438.

[3] E. Kirkman, J. Kuzmanovich. On the global dimension of fibre products. Pacific J. Math., 134 (1988), 121-132.

[4] S. Maclane. Homology. Springer-Verlag, New York, 1963. 
[5] J. MiLnor. Introduction to Algebraic K-theory. Annals Math. Studies, vol. 72, Princeton University Press, Princeton, 1971.

[6] S. Scrivanti. Homological dimension of pullbacks. Math. Scand., $71(1992), 5-15$.

Equipe de Mathématiques

U.M.R. 6623 du C.N.R.S.

16 , route de Gray

25030 Besançon Cedex France

Department of Mathematics and Mechanics

Saint-Petersburg State University

Bibliotechnaya pl. 2

Saint-Petersburg, 198904, Russia

E-Mail: koko@nk1442.spb.edu 